\title{
El nivel de conocimiento y la reputación social de las empresas cooperativas. El caso de la Región de Murcia
}

\section{Federico Martínez-Carrasco Pleite y Maged Eid}

RESUMEN: En este trabajo, partiendo de la información generada con una encuesta realizada a 321 ciudadanos de la Región de Murcia, se cuantifica el grado de conocimiento, la notoriedad, la imagen y la reputación que de las empresas cooperativas tiene su población. La información generada revela el aún insuficiente conocimiento que los ciudadanos tienen acerca de las cooperativas y de la Economía Social. Aún así, se comprueba la positiva imagen que de las mismas se tiene en relación a sus beneficios sociales; en el desarrollo de valores, en la generación de empleo de calidad o por su contribución al desarrollo de los territorios. El estudio de las dimensiones de su reputación empresarial realizado, permitió comprobar un nivel débil o vulnerable en todas ellas, siendo por lo general mayor la valoración que los ciudadanos otorgan a las empresas capitalistas, salvo en dos dimensiones concretas, como son la integridad empresarial o la ciudadanía.

PALABRAS CLAVE: Conocimiento, notoriedad, imagen, reputación, cooperativas, Región de Murcia.

CLAVES ECONLIT: P13, P12, L14, L19, D22, A13.

Cómo citar este artículo / How to cite this article: MARTíNEZ-CARRASCO PLEITE, F. \& EID, M. (2017): "EI nivel de conocimiento y la reputación social de las empresas cooperativas. El caso de la Región de Murcia", CIRIEC-España, Revista de Economía Pública, Social y Cooperativa, 91, 5-29.

Correspondencia: Federico Martínez-Carrasco Pleite, Dpto. de Economía Aplicada, Facultad de Economía y Empresa, Cátedra de Economía Social, Universidad de Murcia. E-mail: femartin@um.es.

Maged Eid, Becario Doctoral MAEC-AECID, Dpto. de Economía Aplicada, Facultad de Economía y Empresa, Universidad de Murcia, maged@um.es. 


\section{EXPANDED ABSTRACT}

\section{The level of knowledge and the social reputation of cooperative companies. The Region of Murcia case}

Objectives. According to the International Labor Organization, one of the main barriers to the development of the cooperative model throughout the world is the lack of knowledge and social awareness about its values and its contribution to development. This study a aims precisely to know the level of knowledge and social awareness that citizens have about the contribution of cooperative enterprises to the development of a more economically and socially responsible economic model, favoring the reduction of poverty, the creation of more stable jobs and that favors social integration. The main objective of this work was to study the level of knowledge that citizens have about the cooperative business model and its inspiring principles. Secondly, to check its reputation level against conventional capitalist companies, approaching their level of excellence based on the value that citizens give to these companies through seven large dimensions of reputation. To achieve this goal, a field study was designed, with which to gather primary information about the impressions, news and values that citizens have about cooperative societies.

Methodology. For the study of the level of knowledge that citizens have about cooperative companies, a questionnaire was designed with 21 questions, which was used with 321 citizens in year 2014 at different locations in the Region of Murcia, located in the southeast of Spain. With this questionnaire, besides approximating to the level of knowledge and the global assessment people have of this type of collective enterprise, also was studied the image that citizens have about these companies. For the study of business reputation, respondents were asked to value companies based on seven dimensions, following the RepTrak Pulse model. The seven dimensions considered were: D1. Quality: it offers excellent products and quality services. D2. Degree of innovation: offers innovative products or services or innovates in the way of doing things. D3. Treatment of employees: the company is an attractive place to work as an employee. D4. Ethics in management: is a company managed responsibly, behaving ethically and shows its operations with transparency. D5 Concern for society: the company cares about society, invests in good causes and does not harm the environment. D6. Leadership: is a wellled company, having recognized leaders and is managed with professionalism. D7. Profitability: it is a profitable company, obtaining good financial results. In addition to calculating a composite indicator of the level of reputation of the companies, the valuations achieved identify the dimensions in which the cooperatives have a higher or lower reputation. 


\section{EL NIVEL DE CONOCIMIENTO Y LA REPUTACIÓN SOCIAL DE LAS EMPRESAS COOPERATIVAS. EL CASO DE LA REGIÓN DE MURCIA}

Results. The extensive first information generated with this survey made it possible to verify how two thirds of the surveyed citizens trust cooperative enterprises. Although the number of people who trust cooperatives is lower than those who trust small and medium enterprises, it is practically twice as many as the number of citizens who trust large companies, which barely reaches a third of the population.

Social Economy concept is scarcely known among citizens, given that it was found that only three out of ten citizens know that involves different types of companies, such as cooperatives, insertion companies or foundations, as established in Spanish Social Economy Law since 2011. In the specific case of cooperatives, almost 8 out of ten citizens know that they are a "specific type of company form", the other respondents not knowing what form of organization this is . In particular, a small percentage thinks they are the same as non-profit entities. The level of knowledge of particular cooperative companies is wide, since 7 out of ten citizens are able to identify and name a cooperative company they know. However, only 15\% knew that in 2012 the International Year of United Nations Cooperatives was celebrated. Social link with the cooperatives was found to be high, seeing as $11 \%$ of the respondents wer -or had been- a member of a cooperative, such as agrarian, consumer, housing, teaching or banking. $45 \%$ indicated that they had a relative or close person who was linked to a cooperative, as a partner, worker or consumer. Then they were asked about three large cooperatives (food distribution and a bank), with a large presence throughout the Spanish territory, only 3 out of ten citizens knew these companies have the legal form of cooperative.

The study of cooperative movement in the Region, continued analyzing the image that the citizens surveyed have about this type of firms. Nearly 7 out of ten highlighted several values that identify these entities: they are more democratic companies; they are as competitive as other companies; they are a type of companies that support and promote values such as collaboration or democracy, clearly differentiating themselves from other types of capitalist companies. However, only one out of two respondents believed that it is a modern business model with a promising future given that it is a more responsible type of company.

The aggregation of the qualifications given by the respondents in seven dimensions of the reputation from cooperatives and from specific capitalist companies, allowed to analyze the global valuation granted each of them. Thus, the level of reputation of the cooperatives is globally slightly lower than that reached by the capitalist companies $(6,1<6,3$ according to a scale of 1 to 10). Both among cooperatives and capitalist companies, are financial entities (Banks) those achieving a lower average reputation, followed by super-hypermarkets, with the highest value been given to cooperatives and capitalist companies of "Other sectors". The capitalist companies achieve a higher valuation in several dimensions: in Profitability (D7); in Quality of the offer (D1); in Leadership (D6); in Degree of innovation (D2); and finally, in Employee Treatment (D3). On the contrary, cooperative firms outperform capitalists in only two dimensions, such as: Ethics in their management (D4), and second, in Concern for society (D5). Although this study has generated ample information about areas for improvement of the coo- 
perative model, it would be necessary to extend this study to other geographical areas, carrying out larger studies that measure the level of reputation and image that this type of company has in Spain and in Europe.

Practical conclusions and original value. In spite of having verified that the citizens of Murcia have an adequate level of knowledge of the cooperative companies, very common in Spain, especially in rural environments, it is still important the educational task that should continue to be carried out in order to let this type of company be better known through the values it promotes and on which it is based, and how cooperative principles allow to develop more responsible and sustainable companies.

The image that citizens have about cooperatives, in comparison with that about capitalist companies, is particularly good in terms of their contribution to the "development of the rural areas", the "internal democracy" and their "professionalism", being able to "compete in equal conditions" with other companies. Although it has been possible to verify that the level of reputation that cooperatives generally have is "Weak" or "Vulnerable", this is better overall than that reached by capitalist companies.

Similarly, dimensions have been identified in which it is necessary for cooperatives to increase their competitive efforts, such as innovation or product quality. The social reputation of cooperatives only stand out in two dimensions, related to a more ethical behavior (Responsibility) and a greater concern for society (Citizenship). On the contrary, there is no greater reputation of cooperatives - in front of the capitalist companies - in other areas, such as their ability to generate quality working conditions, and it is urgent that cooperative enterprises recover the recognition of some of the cooperative principles that identify and differentiate them.

This work provides an unprecedented comparative study of the business reputation of cooperatives versus capitalist companies. The information generated through a survey has allowed to study the reputation of cooperatives, in a total of seven large dimensions, which provide very relevant information about their strengths and weaknesses in the opinion of citizens, and about spaces for competitive ness improvement of the cooperative model.

KEYWORDS: Knowledge, reputation, image, reputation, cooperatives, Region of Murcia. 


\section{EL NIVEL DE CONOCIMIENTO Y LA REPUTACIÓN SOCIAL DE LAS EMPRESAS COOPERATIVAS. EL CASO DE LA REGIÓN DE MURCIA}

\section{Introducción}

En la declaración del "Año Internacional de las Cooperativas" 2012 de la Organización de Naciones Unidas (ONU, 2010)1 ${ }^{1}$, tras señalarse la función que este tipo de empresa tiene en el desarrollo de muchas zonas del mundo, proponía como primero de sus tres grandes objetivos favorecer la creación de una mayor conciencia del público sobre la contribución de estas empresas en el desarrollo económico y social, y de manera especial, en la reducción de la pobreza, la creación de empleos y la integración social. Como señalaba la ONU (2011), para aumentar la conciencia pública sobre las cooperativas y su contribución al desarrollo sostenible, se necesita la colaboración de todas las partes relacionadas o involucradas de manera directa o indirecta en el movimiento cooperativo, en el camino de: a) Promover y compartir una definición clara de las cooperativas y de sus características. b) Destacar las fortalezas de las empresas cooperativas en la promoción del desarrollo sostenible y en las economías. c) Informar sobre la viabilidad del modelo cooperativo, la presencia y el éxito de las cooperativas existentes y sus contribuciones a la innovación social, económica y ambiental. d) Identificar y evaluar las vías y medios para crear conciencia sobre las cooperativas.

Si bien son cada vez más los países que cuentan con estadísticas referentes al número de cooperativas, las personas asociadas o los empleos generados, las informaciones y el conocimiento social acerca del movimiento cooperativo sigue siendo insuficiente. En el caso de España, las estadísticas -del número de cooperativas o del impacto que estas tienen en términos de generación de empleo 0 riqueza- son facilitadas desde el año 1992 por el Ministerio de Trabajo, Empleo y Seguridad Social (MTESS, 2016)². Esa información pone de manifiesto la dimensión que este tipo de empresas tiene dentro del sistema económico nacional y regional, apenas conocido por los ciudadanos.

Tal y como establece la Alianza Cooperativa Internacional (ACI), una cooperativa es "una asociación autónoma de personas unidas voluntariamente para satisfacer sus necesidades y aspiraciones económicas, sociales y culturales comunes a través de una empresa de propiedad conjunta y controlada democráticamente". Este tipo de empresa, que se rige a nivel nacional por la Ley 27/1999, de 16 de julio, de Cooperativas constituye un modelo de empresa que promueve de manera especial la democracia, la solidaridad y la igualdad, conforme a los principios cooperativos en los que se

1.- Más información sobre la declaración de la ONU del Año Internacional de las Cooperativas 2012 en: http://www.un.org/es/events/coopsyear/

2.- Según esa fuente, en el segundo trimestre de 2016 eran 20.524 el número de sociedades cooperativas inscritas en la Seguridad Social en España, que daban empleo a 348.594 personas en todo el territorio nacional (de los que correspondían a la Región de Murcia 2.096 empresas y 24.175 empleados), dando muestras de la importancia que este modelo tiene, que en 2013 alcanzaba un valor total de producción superior a los 44 mil millones de euros en toda España, o 2.649 millones de euros en la Región de Murcia. 
sustenta. Son números los estudios en el ámbito nacional e internacional que han analizado cómo los principios cooperativos le confieren a este tipo de empresa la capacidad de hacer frente a los cambios en el entorno -y promoverlos-, mostrándose más resilientes en tiempos de crisis que las empresas capitalistas (Pérotin, 2012). Se trata de un modelo que promueve organizaciones más participativas en su gobierno y gestión (Marcuello y Saz, 2008), con un sistema de reparto de los beneficios más equitativo, según el grado de contribución y consecución de cada socio (Birchall y Simmons, 2013). Además, los principios cooperativos pueden influir positivamente en la competitividad de las organizaciones (Vargas, 1995; Agirre, 2001; Mozas et al., 2005), aportándoles en algunas ocasiones determinadas ventajas competitivas (Moreira, 1999). El principio de interés por la comunidad permite a las cooperativas interactuar con su entorno siguiendo los valores de la cooperación y la solidaridad que, a diferencia de los valores de individualismo actuales -propios del modelo capitalista-, promueven la inclusión y la justicia social (Defourny y Nyssens, 2012). Promueven un espíritu comunitario, lo que permite una mayor implicación y participación de los individuos en el desarrollo local (Lais, 2009), base del desarrollo sostenible (Vidal, 2008). Por lo tanto, los principios cooperativos, inspiradores de este modelo organizativo, constituyen el elemento diferencial de este tipo de empresas, con beneficios sociales no suficientemente reconocidos.

Pese a la dimensión e importancia que el modelo cooperativo alcanza en el ámbito internacional, la Organización Internacional del Trabajo (OIT, 2012) recordaba que "el desarrollo de las cooperativas está impedido por la falta de conocimiento y comprensión del modelo de la empresa cooperativa", siendo precisamente ese el objeto de estudio de este trabajo. En los próximos apartados se presentan los resultados de una encuesta realizada a ciudadanos de la Región de Murcia, con la que conocer el grado de conocimiento que de estas empresas tiene la población de ese ámbito geográfico, analizándose su nivel de reputación social frente a otros tipos de empresas.

\section{Antecedentes}

Son cada vez más las investigaciones que analizan la valoración que de las empresas -y de sus productos- tienen los consumidores, siendo relevante la revisión teórica que del concepto de reputación empresarial realizan Martínez y Olmedo (2010). Según Fomburn (1996) es "una percepción representativa de la compañía, basada en su actuación en el pasado así como en su proyección futura". Groenland (2002) lo describe como un concepto emocional, difícil de racionalizar y de expresar en palabras. Según Sanz y González (2005), la reputación es el reconocimiento valorativo que los públicos interesados otorgan a la empresa. Pérez y Rodríguez del Bosque (2014) aúnan todas esas definiciones de la siguiente manera: "la fotografía mental que reconcilia las múltiples imágenes que de una empresa poseen varias audiencias, y que se construye y desarrolla a lo largo del tiempo". Un 


\section{EL NIVEL DE CONOCIMIENTO Y LA REPUTACIÓN SOCIAL DE LAS EMPRESAS COOPERATIVAS. EL CASO DE LA REGIÓN DE MURCIA}

ejemplo de medición de la valoración que determinados grupos de interés dan a empresas sociales lo encontramos en el trabajo de Martínez et al. (2013), que analizan la reputación percibida por el profesorado de cooperativas de trabajo asociado en el ámbito de la educación, y el impacto que su satisfacción laboral tiene sobre la calidad del servicio. De manera más amplia, un referente indispensable en el estudio del nivel de conocimiento y valoración social de las cooperativas en España es el trabajo que, para el caso de la Comunidad Valenciana, realizó la Confederación de Cooperativas de la Comunidad Valenciana en los años 2000 y 2007, titulado la "Imagen del cooperativismo en la Comunidad Valenciana" (CONCOVAL, 2008).

La reputación empresarial depende de los grupos de interés, siendo un activo que les permite crear valor (Borraz y Fuentelsaz, 2005) y generar beneficios empresariales (Wessels, 2003; Villafañe, 2004), como por ejemplo mediante la reducción de los costes de transacción y de asimetrías en la información; también permite la contratación de trabajadores mejor preparados, o el acceso más favorable al mercado de capitales; por tanto, permite crear barreras de entrada a la competencia y por consiguiente elevar los precios de venta (Martínez y Olmedo, 2010). Conviene recordar que una vez construida la reputación, hace falta consolidarla y preservarla, a través del desarrollo y del mantenimiento de un mayor compromiso público en cuanto a los derechos humanos, laborales, medioambientales y de reducción de prácticas de corrupción.

Más recientemente Pérez y Rodríguez del Bosque (2014) plantean una revisión integradora de diferentes enfoques entorno a los conceptos identidad, imagen y reputación de la empresa, que les lleva a aportar la siguiente distinción: la identidad de una empresa se refiere al "carácter central, distintivo y duradero de la empresa, bajo control de esta, y que por tanto, corresponde a un concepto de carácter interno a la organización"; por su parte, la imagen se refiere "a la percepción puntual de la empresa que se deriva de la suma de informaciones, impresiones, expectativas, creencias y sentimientos que un individuo acumula sobre ella", haciendo por tanto mención a la percepción de los individuos como consecuencia y resultado de las acciones corporativas. Finalmente, "la suma de las imágenes individuales acumuladas por los grupos de interés configura la reputación de la empresa, entendida ésta como una percepción global de la empresa por parte de todas las audiencias de la misma y que, además, se gestiona a lo largo del tiempo, de tal manera que es estable y relativamente continua en el tiempo". La reputación sería por tanto la agrupación del análisis de las visiones interna y externa, de todos los grupos, desarrollada a lo largo del tiempo, y fruto de un proceso complejo de interacciones.

En la última década son varias las instituciones que han propuesto metodologías e índices sintéticos de medición de la reputación empresarial (Mandelli y Cantoni, 2010; Orozco y Ferré, 2012)33, con el objetivo de valorar aspectos intangibles y los resultados de estrategias de comunicación. De entre

3.- Fortune ("The World's Most Admired Companies"); Financial Times (FT Global 500 "The world's largest companies"); The Harris Poll Reputation Quotient (RQ); Dow Jones Sustainability Index (DJSI); Ipsos, Key Audience Reserch (KAR); Monitor Empresarial de Reputación Corporativa (Merco); y Foro de Reputación Corporativa (RepTrack Pulse). 
las aplicaciones existentes en el caso de España (Ipsos, Merco y RepTrak Pulse), resulta de especial interés la última de las opciones mencionadas, realizada por el Reputation Institute (2013), y que será aplicada en este trabajo. A través de encuestas, recopila datos sobre las percepciones de las partes interesadas, construyendo un indicador llamado RepTrak Pulse basado en esos elementos: admiración y respeto, reputación reconocida, buena impresión y confianza, que son una serie de sentimientos que tienen las personas hacia la empresa, incluidos en el cuestionario en siete grandes dimensiones que forman el modelo. Con esa información se elabora el Índice Global de Reputación, ofreciendo cada año un ranking de las empresas mejor valoradas en España, que en 2013 era liderado por Danone; Sony; Google; B/S/H; Nestlé; BMW; Volkswagen; Daimler; Decathlon; Michelin (Reputation Institute, 2014), apareciendo dos cooperativas en ese listado: una era la cadena de distribución Eroski, que ocupaba la posición número 62 de ese listado; la segunda, ocupando la posición 100, era la cadena de supermercados Consum.

Partiendo de la hipótesis de que las empresas cooperativas cumplen y se rigen por los principios cooperativos, se entiende que este modelo de empresa pudiera contar con una estructura interna y un funcionamiento externo consolidados con los que poder adquirir mayor reputación empresarial frente a otros tipos de empresas (Olmedo et al., 2012), siendo uno de los objetivos de este estudio comprobar cuál es su nivel de reputación, como "suma de informaciones, impresiones, expectativas, creencias o sentimientos que cada individuo acumula" (Pérez y Rodríguez del Bosque, 2014).

\section{Metodología}

Los datos que se presentan en los siguientes apartados proceden de una encuesta que se realizó en 2014 en distintas localidades de la Región de Murcia, tras la finalización del "Año Internacional de las Cooperativas 2012" de la ONU y la proclamación -a final de ese año- del Proyecto "Desafío 2020" de la Alianza Cooperativa Internacional (ACl, 2013; Eid y Martínez-Carrasco, 2014). La encuesta se realizó a 321 ciudadanos mayores de edad residentes en la Región de Murcia, siguiendo un muestreo aleatorio estratificado (atendiendo la distribución por sexos, edades y localización geográfica de la población regional), que determina un error muestral del $\pm 4,24 \%$ en proporciones intermedias y del $\pm 7,07 \%$ en extremas, válido en este tipo de estudios. El cuestionario, tras someterse a una prueba piloto con una veintena de personas, contaba con 21 preguntas incluidas en tres grandes apartados:

Bloque I.- Perfil del encuestado. Este incluía un grupo de variables de caracterización socio-demográfica de los encuestados, que posibilitó un posterior análisis de relación bivariante, empleándose el paquete estadístico SPSS-19. 


\section{EL NIVEL DE CONOCIMIENTO Y LA REPUTACIÓN SOCIAL DE LAS EMPRESAS COOPERATIVAS. EL CASO DE LA REGIÓN DE MURCIA}

Bloque II.- Conocimiento y notoriedad de cooperativas. Este contenía preguntas acerca del conocimiento de los ciudadanos de la Economía Social, y de manera muy especial de las cooperativas, contando con una pregunta con la que recabar los nombres de cooperativas, con el que estudiar la notoriedad 4 de estas. Este apartado continuaba con una serie de preguntas con las que recabar: primero, el conocimiento o no de la forma jurídica que tienen grandes cooperativas presentes en la Región de Murcia (Eroski, Cajamar o Consum); y segundo, la valoración que otorgaban a una serie de afirmaciones (su nivel de acuerdo o no con las mismas se establecía a través de una escala de Likert de 1 a 5) sobre aspectos diferenciales de las cooperativas en relación a otras empresas mercantiles referentes a su mayor compromiso y responsabilidad, empleadas previamente por $\mathrm{CON}$ COVAL (2008).

Bloque III.- Nivel de reputación de las cooperativas. Tenía por objeto identificar las empresas más valoradas por los ciudadanos, siguiendo el modelo propuesto por Reputation Institute (2013). Lo primero, era preguntarles qué dos empresas quería comparar, enfrentando a una cooperativa con una empresa convencional, debiendo en ambos casos garantizarse un "nivel de conocimiento mínimo de la empresa por cada entrevistado". En segundo lugar se les planteaba una primera afirmación a valorar ("genera un alto nivel de confianza, respeto, admiración, estima o buena impresión") con la que aproximar el nivel de reputación emocional. En tercer lugar, se les pedía a los encuestados que valorasen las empresas en siete dimensiones, incluyéndose una descripción de cada una de ellas: D1. Calidad de la oferta: "ofrece productos excelentes y servicios de calidad". D2. Grado de innovación: "ofrece productos o servicios novedosos o bien innova en la manera de hacer las cosas". D3. Trato de empleados: "la empresa es un lugar atractivo para trabajar como empleado". D4. Ética en la gestión: "es una empresa gestionada con responsabilidad; se comporta de forma ética y muestra su operativa con transparencia". D5. Preocupación por la sociedad: "la empresa se preocupa por la sociedad, invierte en buenas causas y no daña el medio ambiente". D6. Liderazgo: "es una compañía bien liderada, teniendo líderes reconocidos y está gestionada con profesionalidad". D7. Rentabilidad: "es una compañía rentable, obteniendo buenos resultados financieros". Finalmente, la media aritmética de las valoraciones obtenidas en cada dimensión dio lugar al Índice Global de Reputación ${ }^{5}$, tal y como se describe en el último de los epígrafes.

4.- La notoriedad, entendida como la capacidad de los consumidores de reconocer, recordar o recomendar (Rossiter y Percy, 1987), es de fácil aproximación, comprobando el número de personas que conocen marcas, productos o la propia empresa (Alameda et al., 2006).

5.- La valoraciones globales o parciales de cada dimensión -que se restringió a un valor máximo de 10 por su facilidad de respuesta y simplicidad de interpretación- son objeto de comentario en el último de los apartados de resultados, atendiendo a la siguiente escala de calificación del nivel de reputación alcanzado: Excelente o superior (>8); Fuerte o robusta (7-7,9); Media o moderada (6-6,9); Débil o vulnerable (4-5,9); Débil o pobre (<4). 


\section{Resultados}

Los datos obtenidos con la encuesta se procuró se ajustasen lo máximo posible a las características socio-demográficas de la población de la Región de Murcia. Así, el perfil de la muestra alcanzada responde a que un $51 \%$ de los encuestados eran hombres y el resto mujeres; por edad, el promedio era de 42,5 años, habiéndose categorizado los encuestados en tres grupos: jóvenes (de 18 a 34 años), adultos (de 35 a 49 años) y mayores (>50 años). En cuanto al nivel de estudios obtenidos, el $59 \%$ de la muestra contaba con un nivel de estudios inferior, igual o equivalente a Bachiller/FP. En cuanto al nivel de Renta familiar, el 78\% de los encuestados tenía un nivel de renta menor a 2.700 euros por mes, que atendiendo al tamaño familiar medio determinaba una renta per cápita de 616 $€ /$ mes. Un $52 \%$ de los encuestados eran residentes en grandes ciudades de la Región de Murcia (con más de 90 mil personas), repartiéndose el resto de la muestra -en dos grupos de casi igual tamañoentre residentes de ciudades medianas (90-30 mil) y pequeñas (menos de 30 mil habitantes).

\subsection{Confianza en las cooperativas y conocimiento de la Economía Social}

El cuestionario comenzaba comprobando el nivel de confianza que los ciudadanos otorgan a las empresas cooperativas, así como a otras organizaciones e instituciones como son las Pequeñas y Medianas Empresas (PYMEs), las ONGs (Organizaciones No Gubernamentales) o la Justicia. En las primeras columnas del Cuadro 1 se ofrecen los resultados obtenidos con la encuesta, mostrándose de manera complementaria las valoraciones que cuatro años antes alcanzaban ${ }^{6}$ esos actores en España (Comisión Europea, 2010). Tal y como puede comprobarse en el Cuadro 1, los ciudadanos murcianos -tal y como a nivel nacional sucedía- muestran su máximo nivel de confianza hacia PYMEs (el 75\% "confían"), siendo las instituciones hacia las que muestran sus mayores niveles de "desconfianza": los Partidos políticos, los Sindicatos y el Gobierno. La incorporación de las cooperativas entre las instituciones a valorar, permite comprobar cómo en estas confían un muy alto porcentaje de ciudadanos $(65,7 \%)$, que si bien no llega a ser el que logran las PYMEs, se encuentra diez puntos por encima del que alcanzan las ONGs.

6. - Si bien la distancia en el tiempo de esos datos imposibilita una comparación adecuada con las valoraciones ofrecidas por esa fuente oficial, se entendió de interés ofrecerlos por aportar: una referencia nacional de la valoración social realizada, además de información de la posible evolución temporal de los niveles de confianza hacia determinadas instituciones. 

EL NIVEL DE CONOCIMIENTO Y LA REPUTACIÓN SOCIAL DE LAS EMPRESAS COOPERATIVAS.
EL CASO DE LA REGIÓN DE MURCIA

\section{Cuadro 1. Confianza ciudadana en las cooperativas y en otras entidades}

\begin{tabular}{|c|c|c|c|c|c|c|c|}
\hline \multirow{2}{*}{$\begin{array}{l}\text { FR (\%). } \\
\text { Ranking Empresas }\end{array}$} & \multicolumn{3}{|c|}{ Región de Murcia* } & \multicolumn{3}{|c|}{ España $^{\star \star}$} & \multirow{2}{*}{ Total } \\
\hline & Confían & Desconfían & $\mathrm{Ns} / \mathrm{Nc}$ & Confían & Desconfían & $\mathrm{Ns} / \mathrm{Nc}$ & \\
\hline $1^{\circ}$ PYMEs & 75,07 & 11,53 & 13,40 & 71 & 20 & 9 & 100 \\
\hline $2^{\circ}$ Cooperativas & 65,74 & 15,26 & 19,00 & $\mathrm{Nd}$ & $\mathrm{Nd}$ & $\mathrm{Nd}$ & $\mathrm{Nd}$ \\
\hline $3^{\circ}$ ONGs & 54,83 & 26,79 & 18,38 & 61 & 27 & 11 & 100 \\
\hline $4^{\circ}$ Grandes empresas & 30,53 & 55,45 & 14,02 & 32 & 55 & 13 & 100 \\
\hline $5^{\circ}$ Justicia & 26,17 & 63,55 & 10,28 & 44 & 51 & 5 & 100 \\
\hline $6^{\circ}$ Gobierno español & 12,77 & 81,00 & 6,23 & 21 & 74 & 5 & 100 \\
\hline $7^{\circ}$ Sindicatos & 11,22 & 79,75 & 9,03 & 30 & 59 & 11 & 100 \\
\hline $8^{\circ}$ Partidos políticos & 2,81 & 90,65 & 6,54 & 5 & 94 & 1 & 100 \\
\hline
\end{tabular}

FR (\%). Frecuencia Relativa, en porcentaje. Ns/Nc: No Sabe o No Contesta. Nd: No disponible.

FUENTE: *Elaboración propia a partir de la Encuesta. ${ }^{*}$ Datos de la Comisión Europa (2010).

A continuación se planteaba a los encuestados dos preguntas relativas a la Economía Social (ES), en cuya familia de entidades ocupan una posición destacada las cooperativas, tal y como establece la Ley 5/2011, de 29 de marzo, de Economía Social. Como puede observarse en el Cuadro 2, tan sólo el $27 \%$ de los encuestados sabía qué era la ES, de los que un $61 \%$ eran hombres y tan sólo el $39 \%$ mujeres. La relación entre el nivel de conocimiento de la ES y el sexo de los ciudadanos resultó ser significativa $\left(X^{2}=4,614 p=0,032\right)$, de igual manera que cuanto más alto es el nivel de formación de los ciudadanos, más elevado es el conocimiento del término $E S\left(X^{2}=25,774 p=0,000\right)$. De entre aquellos que indicaron conocer ese concepto, menos de dos terceras partes supieron dar una descripción acertada del mismo, aportando descripciones válidas -en mayor o menor medida-, describiéndose a continuación algunas: "actividades económicas que de forma privada ejercen entidades, las cuales persiguen intereses colectivos tanto económicos como sociales"; "empresas que trabajan para el desarrollo económico y social"; "actividades económicas que persiguen el bien colectivo de sus integrantes". También fueron frecuentes los encuestados que señalaban en su descripción algunos de sus fundamentos o principios, tales como "la cooperación", "la democracia", "la solidaridad", o "la primacía del trabajo sobre el capital". Entre las respuestas incorrectas, fueron frecuentes los que lo identificaban únicamente con el "Tercer Sector" y las "ONG", indicando que eran "Organizaciones Sin Ánimo de Lucro"; o "empresas cuyos beneficios se destinan a fundaciones de interés social". 


\section{Cuadro 2. Conocimiento de la Economía Social y de sus Federaciones}

\begin{tabular}{|l|cc|c|}
\hline FR (\%) & Sí & No & Total \\
\hline Conocimiento del concepto Economía Social & 27,10 & 72,90 & 100,00 \\
Conocimiento de alguna Federación del sector & 12,15 & 87,85 & 100,00 \\
\hline
\end{tabular}

FUENTE: Elaboración propia a partir de la Encuesta.

Tan sólo un 12 \% la muestra sabía mencionar alguna organización representativa del sector de la ES en España o en la Región de Murcia. Un 69\% de ese porcentaje eran hombres, mostrándose nuevamente una relación significativa entre el conocimiento de alguna Federación y el sexo de los encuestados $\left(X^{2}=5,846 p=0,016\right)$, así como también sucede a medida que aumenta el nivel de estudios. La organización más mencionada (9 ocasiones) fue la Federación de Cooperativas Agrarias de Murcia (FECOAM), seguida por la Asociación de Empresas de Economía Social de la Región de Murcia (AMUSAL) y la Unión de Cooperativas de Trabajo Asociado de la Región de Murcia UCOMUR (5 veces cada una), siendo en tan sólo dos ocasiones mencionadas la Federación de Sociedades Agrarias Cooperativas de Murcia (FECAMUR), así como la Confederación Empresarial Española de la Economía Social (CEPES).

\subsection{Conocimiento y notoriedad de las cooperativas}

El estudio del conocimiento de las cooperativas comenzaba preguntándoles a los encuestados qué eran, siendo tres las opciones posibles (ver Cuadro 3), siguiendo los planteamientos de la CONCAVAL (2008): primero, la opción de indicar no conocer qué es una cooperativa, que fue elegida por el $12 \%$ de los ciudadanos de la muestra; segundo, que son un "tipo de empresa", siendo esta la respuesta mayoritariamente elegida (82\%); y la tercera, que son un "tipo de empresa no lucrativa", elegida por el $6,5 \%$ de los ciudadanos. Estos resultados difieren con los aportados para los ciudadanos de la Comunidad de Valencia en 2007 , con un menor porcentaje (76,1\%) que sabía que la cooperativa es un tipo de empresa, siendo considerablemente superior el porcentaje que según ese otro estudio indicaba que se trataba de organizaciones no lucrativas $(18,5 \%)$. Las diferencias en los resultados de ambos estudios -al margen de diferencias territoriales que se escapaban a los objetivos y a los recursos disponibles para la realización de este trabajo-, pudieran estar mostrando cómo, con el paso de los años es mayor el conocimiento en España del modelo cooperativo, desvinculándose estas del calificativo de "entidades sin fines de lucro". Apenas un 6,5\% indicaron haber escuchado o saber algo de la celebración del Año Internacional de Cooperativas. A la pregunta de cuál había sido el objetivo de la misma, fueron muy escasas las respuestas aportadas, si bien algunas de ellas mostraban un muy acertado conocimiento por parte de esos ciudadanos, tal y como se detalla a continuación: "fomentar el cooperativismo a nivel mundial", "promocionar el mismo" o "concienciar sobre sus beneficios sobre la sociedad, generando empleo e inclusión social". 

EL NIVEL DE CONOCIMIENTO Y LA REPUTACIÓN SOCIAL DE LAS EMPRESAS COOPERATIVAS.
EL CASO DE LA REGIÓN DE MURCIA

\section{Cuadro 3. Conocimiento de las cooperativas entre los ciudadanos}

\begin{tabular}{|l|l|r|}
\hline FR (\%) & & Total \\
\hline Conocimiento del modelo cooperativo & Sí, son un tipo de empresa & 81,93 \\
& Sí, son organizaciones no lucrativas & 6,54 \\
& No, no sé & 11,53 \\
\hline Ha visto o escuchado noticias sobre cooperativas & Sí & 78,82 \\
& No & 21,18 \\
\hline Conocimiento de alguna cooperativa & Sí & 70,09 \\
& No & 29,91 \\
\hline Conocimiento de la celebración en 2012 del "Año Internacional & Sí & 14,02 \\
de las Cooperativas" (AlC-2012) & No & 85,98 \\
\hline Total & & 100,00 \\
\hline
\end{tabular}

FUENTE: Elaboración propia a partir de la Encuesta.

Por el contrario, sí es elevado el porcentaje de ciudadanos (79\%) que decían haber visto, leído o escuchado en algún medio de comunicación alguna noticia de cooperativas, siendo los medios señalados la televisión (40\%), seguida de la prensa escrita (27\%), la radio $(28 \%)$, con un $5 \%$ que indicó haberlo leído en internet. Cabe recordar que en el caso de la Región de Murcia su periódico regional de mayor tirada ( La Verdad) incluye un día a la semana un bloque temático dedicado al mundo cooperativo, con noticias del sector, habiendo existido en la televisión autonómica un programa específico durante varios años destinado al fomento del modelo, con lo que la presencia en los medios e impacto de las noticias del sector pueda ser posiblemente superior en esta Comunidad que en el conjunto nacional.

A continuación, se les preguntaba a los encuestados que indicasen si podían nombrar una cooperativa que recordasen. El nivel de respuesta a esta pregunta fue del $70 \%$, muy superior al alcanzado por el estudio previo realizado hace años en la Comunidad Valenciana (CONCOVAL, 2008), según el cual tan sólo un 38,8\% de los ciudadanos sabía el nombre de alguna cooperativa. Siguiendo con esa pregunta, se les pedía que mencionasen el nombre concreto de alguna cooperativa, a modo de aproximación al nivel de "notoriedad espontánea" de los ciudadanos. Se obtuvo 202 respuestas correctas (un $62,93 \%$ de la muestra), con un $36 \%$ que mencionaron dos y más, y un $27 \%$ de los casos en los que el encuestado mencionó una sola cooperativa. Entre las cooperativas mencionadas en más de 5 ocasiones, destaca el caso de la cadena de hipermercados con presencia en Murcia Eroski (en 32 ocasiones fue mencionada); la cooperativa agroalimentaria regional Coato (29); el supermercado Consum (27); la entidad financiera Cajamar (20); el grupo Mondragón (19); las cooperativas agrarias murcianas Vega del Pliego (14) y Frutas Caravaca (10); el grupo de industrial Fagor (9); la cooperativa agraria regional Cobuco (8); las cooperativas murcianas Bodegas La Purísima (8) y Bodegas San Isidro (7); las cooperativas hortofrutícolas regionales Coáguilas (7) y Alimer (6), Thader Cieza (5) 0 la de producción de aceite de oliva Frusemur (5 veces). 


\subsection{Vínculos de los ciudadanos con las cooperativas}

En cuanto a la pertenencia de los encuestados como socios de alguna sociedad cooperativa, solamente un 10,90\% de la muestra ofreció una respuesta afirmativa (Cuadro 4). Los casos más frecuentemente mencionados de pertenencia como socio (en el presente 0 en algún momento previo) a una cooperativa fueron el caso de la entidad financiera Cajamar -con gran presencia en la Región de Murcia-; de dos de las cooperativas agroalimentarias con un mayor número de asociados, como son Coato y Alimer; siendo también numerosos los que indicaron haber pertenecido a una cooperativa de vivienda.

\section{Cuadro 4. Vinculación de los ciudadanos con las cooperativas}

\begin{tabular}{|c|c|c|c|c|c|}
\hline FR (\%) & & Total & FR (\%) & & Total \\
\hline \multirow[t]{2}{*}{ Socio de alguna cooperativa } & No & 89,10 & \multirow{3}{*}{$\begin{array}{l}\text { Grado de parentesco } \\
\text { o relación con algún socio }\end{array}$} & Si, familiares & 44,07 \\
\hline & Sí & 10,90 & & $\mathrm{Si}$, amigos & 45,76 \\
\hline \multirow{5}{*}{$\begin{array}{l}\text { Familiar o conocido de algún } \\
\text { socio o trabajador de una cooperativa }\end{array}$} & No & 54,52 & & Si, vecinos & 10,17 \\
\hline & Sí & 45,48 & \multirow{4}{*}{$\begin{array}{l}\text { Consumidor de productos } \\
\text { de cooperativas }\end{array}$} & No & 40,82 \\
\hline & -Trabaja & 33,67 & & $\mathrm{Si}$ & 50,47 \\
\hline & -Socio & 39,80 & & $\mathrm{Ns} / \mathrm{Nc}$ & 8,41 \\
\hline & -Ambos & 26,53 & & & \\
\hline Total & & 100,00 & Total & & 100,00 \\
\hline
\end{tabular}

FUENTE: Elaboración propia a partir de la Encuesta.

Relacionado con el entorno del encuestado, y su conocimiento de alguna persona cercana socia de una cooperativa, el $54 \%$ no recordaban o sabían de ningún familiar, amigo o vecino que trabajara - o fuese socio- de una cooperativa. Por el contrario, un $45 \%$ de la muestra afirmaba conocer a alguna persona que sí lo era, que trabajaba en alguna cooperativa, o que además era socia. Por último se le preguntaba a los encuestados si sabían de algún producto o servicio por ellos consumido, que era producido o vendido por alguna cooperativa. Algo más de la mitad de los ciudadanos $(50,5 \%)$ afirmó haber consumido en algún momento algún producto o servicio procedente de una cooperativa, siendo los productos de alimentación y bebidas los más frecuentemente mencionados.

Para recordar la importancia social que el movimiento cooperativo alcanza en la Región de Murcia, baste señalar que se estima que son más de 20.000 los agricultores -a tiempo parcial o no- que están asociados a cooperativas agrarias de FECOAM (un 1,4\% del total de un millón cuatrocientos mil murcianos). A esos habría que sumar otros cerca de 5.000 productores integrados en cooperativas de FECAMUR, no incluyendo en ambas cifras los empleos directos e indirectos ligados a esos agricultores cooperativistas. Su importancia en el ámbito local puede llegar a ser muy elevado en muchas localidades rurales, siendo uno de los ejemplos más llamativos el caso de la cooperativa COATO, inte- 


\section{EL NIVEL DE CONOCIMIENTO Y LA REPUTACIÓN SOCIAL DE LAS EMPRESAS COOPERATIVAS. EL CASO DE LA REGIÓN DE MURCIA}

grada por 3.500 agricultores, cuyas instalaciones se sitúan en un municipio (Totana) de cerca de 30.000 habitantes. La vinculación e impacto del cooperativismo en la sociedad murciana queda de manifiesto nuevamente en el caso de las cooperativas de enseñanza, que contando con algo menos de 80 centros de formación, da cobertura formativa a un importante número de familias murcianas, con más de 32.000 niños y jóvenes en ellas inscritos, dando empleo a más de tres mil personas, asociadas en su mayor parte (Martínez-Carrasco, 2011). La escasez de más estadísticas acerca de la importancia e impacto del sector cooperativo, impide aportar una aproximación más detallada de los vínculos de la ciudanía con el modelo cooperativo.

\subsection{Imagen y beneficios de las cooperativas}

El cuestionario contaba con una ficha informativa en la que, tras preguntarles sobre su conocimiento y vinculación con las cooperativas, se les mostraba una breve descripción de qué es una cooperativa. Así, y tras explicarles que las cooperativas "son empresas en las que sus socios, son propietarios y participan de manera democrática en la toma de decisiones bajo el principio de una persona es un voto, participando en el reparto de beneficios y en la gestión, siendo habituales en el ámbito agrario pero también en otros sectores", se daba paso a otras preguntas. Se comenzaba preguntándoles, a modo de estudio del nivel de notoriedad -en este caso "inducida o asistida"-, si sabían que el hipermercado Eroski, el supermercado Consum, o la caja de ahorros Cajamar, eran cooperativas. Los resultados llevaron a comprobar que tan sólo el 38\% de los ciudadanos sabían que Eroski es una cooperativa; al igual que pasaba para un $36 \%$ en el caso Cajamar, o en un $30 \%$ para Consum, todas ellas con una amplia presencia en la Región de Murcia.

El cuestionario planteaba a continuación una pregunta que contenía siete afirmaciones relacionadas con la imagen de las cooperativas y sus beneficios en términos de calidad en el empleo y promoción de valores, empleado por la CONCOVAL (2008). Tal y como se observa en el Cuadro 5, fue máximo el nivel de acuerdo entre los encuestados (70\% estaban de acuerdo o muy de acuerdo) con la afirmación en la que se indicaba que las cooperativas "son empresas más democráticas..." (A4), con un $67 \%$ que entendía que "contribuyen muy positivamente al desarrollo económico de las localidades donde se sitúan..." (A3). El nivel de competitividad de las cooperativas era respaldado por la opinión de los ciudadanos, por cuanto un $65 \%$ de la muestra entendía "que trabajan de manera profesional, compitiendo perfectamente con otras empresas privadas" (A6), reconociéndole a las cooperativas un $64 \%$ de la muestra que "son un modelo de empresa que se sustenta y promueve valores (colaboración, democracia, etc.), diferenciándose claramente de otros tipos de empresas" (A1). En el resto de afirmaciones el nivel de consenso es ligeramente inferior. Tal era el caso de la afirmación que hacía referencia a que las cooperativas "mantienen un enfoque más responsable" (A5), o que se trata de un modelo de empresa "actual y moderna, con un futuro prometedor por cuanto se trata de empresas con más valores y más responsables socialmente" (A7), con las que un $53 \%$ de los ciudadanos estaban parcial o totalmente de acuerdo. Por último, la afirmación menos valorada era la que señalaba cómo las cooperativas "generan empleo más estable y de más calidad, siendo más resistentes 
a las crisis, no estando sujetas a procesos de deslocalización como las multinacionales" (A2); si bien un $47 \%$ de los ciudadanos estaban de acuerdo, los niveles de desacuerdo eran máximos, con un $13 \%$ de la población que no consideraba fuese cierta esa consideración.

\section{Cuadro 5. Afirmaciones acerca de la imagen de las cooperativas}

\begin{tabular}{|c|c|c|c|c|c|c|c|}
\hline \multirow{2}{*}{$\begin{array}{l}\text { Valoración de } 1 \text { a } 5 \text { siendo } \\
\text { (1 en total desacuerdo y } 5: \text { Totalmente de acuerdo) }\end{array}$} & \multicolumn{4}{|c|}{ FR (\%) } & \multirow{2}{*}{$\mathrm{Me}$} & \multirow{2}{*}{ Md } & \multirow{2}{*}{ Mo } \\
\hline & $1+2$ & 3 & $4+5$ & $\mathrm{NS}^{*}$ & & & \\
\hline $\begin{array}{l}\text { A1. Las cooperativas son un "modelo de empresa" que } \\
\text { se sustenta y promueve valores (colaboración, democracia,...), } \\
\text { diferenciándose claramente de otros tipos de empresas. }\end{array}$ & 6,85 & 19,63 & 64,18 & 9,35 & 4,15 & 4 & 4 \\
\hline $\begin{array}{l}\text { A2. Las cooperativas generan empleo más estable y de más } \\
\text { calidad, siendo más resistentes a las crisis, no estando sujetas } \\
\text { a procesos de deslocalización como las multinacionales. }\end{array}$ & 13,4 & 25,86 & 47,04 & 13,71 & 3,91 & 4 & 4 \\
\hline $\begin{array}{l}\text { A3. Las cooperativas contribuyen muy positivamente al } \\
\text { desarrollo económico de las localidades donde se sitúan, } \\
\text { siendo muy importantes en el ámbito rural. }\end{array}$ & 5,61 & 13,08 & 66,67 & 14,64 & 4,41 & 5 & 5 \\
\hline $\begin{array}{l}\text { A4. Las cooperativas son empresas más democráticas, } \\
\text { compartiendo las decisiones los socios }\end{array}$ & 5,92 & 12,77 & 70,41 & 10,90 & 4,33 & 4 & 5 \\
\hline $\begin{array}{l}\text { A5. Las cooperativas mantienen un enfoque más responsable, } \\
\text { al no solo tener en cuenta los resultados, si no también lo social } \\
\text { y el desarrollo local. }\end{array}$ & 9,34 & 21,18 & 52,65 & 16,82 & 4,13 & 4 & 4 \\
\hline $\begin{array}{l}\text { A6. Las cooperativas son empresas que trabajan de manera } \\
\text { profesional, compitiendo perfectamente con otras empresas } \\
\text { privadas. }\end{array}$ & 7,16 & 15,26 & 64,80 & 12,77 & 4,27 & 4 & 5 \\
\hline $\begin{array}{l}\text { A7. Se trata de un modelo de empresa "actual y moderna", } \\
\text { con un "futuro prometedor", por cuanto se trata de empresas } \\
\text { con más valores y más responsables socialmente. }\end{array}$ & 10,90 & 20,56 & 52,65 & 15,89 & 4,09 & 4 & 4 \\
\hline
\end{tabular}

Nota: Si bien se ofrece el valor Medio (Me) de las respuestas, la Mediana (Md) y la Moda (Mo) aportan una muy adecuada aproximación como medidas de centralidad de las respuestas, por lo que se decidió incluirlas.

FUENTE: Elaboración propia a partir de la Encuesta.

\subsection{Empresas con mayor nivel de reputación emocional}

El estudio de la reputación empresarial comenzó con una pregunta en la que se les pedía a los encuestados que indicasen los nombres de alguna empresa o cooperativa (extranjeras, nacionales, regionales o locales), importantes o significativas que pudieran conocer. Se les pedía que las valorasen según una escala de 1 a 10 atendiendo a "la confianza, respeto, admiración, estima o buena impresión" que hacia las mismas tenían (Cuadro 6). Esta pregunta, daba la oportunidad a que cada 


\section{EL NIVEL DE CONOCIMIENTO Y LA REPUTACIÓN SOCIAL DE LAS EMPRESAS COOPERATIVAS. EL CASO DE LA REGIÓN DE MURCIA}

encuestado valorase la empresa que estimase más importante -o significativa-, identificándose de ese modo un listado de las empresas con más notoriedad; y segundo, comprobándose su nivel de reputación "emocional" (Reputation Institute, 2014). Así, fueron cuatro las empresas renombradas en más de cincuenta ocasiones, siendo El Pozo la más frecuentemente mencionada, seguida de Eroski, Mercadona y Cajamar. Atendiendo a la valoración que estas alcanzan, sólo El Pozo y Mercadona contarían con una reputación "Fuerte o robusta" (equivalentes a puntuaciones entre 7 y 7,9), siendo el nivel de reputación de Eroski y Cajamar "Media o moderada". Con un nivel de reputación "Excelente o superior" (puntuación >8) sólo se encontrarían tres empresas (Hero, Estrella de Levante y Coato).

\section{Cuadro 6. Empresas más renombradas por los encuestados (Top 15)}

\begin{tabular}{|l|l|ccccc|}
\hline \multirow{2}{*}{ Menciones } & \multirow{2}{*}{ Nombre Empresa } & \multicolumn{5}{|c|}{ Valoración de 1 a 10 } \\
\cline { 3 - 6 } & & Promedio* $^{*}$ & Mediana & Max. & Min. & Dv \\
\hline 128 & El Pozo & 7,68 & 8 & 10 & 1 & 1,612 \\
86 & Eroski & 6,72 & 7 & 10 & 1 & 1,865 \\
57 & Mercadona & 7,16 & 8 & 10 & 1 & 2,122 \\
51 & Cajamar & 6,57 & 7 & 10 & 1 & 1,993 \\
35 & Hero & 8,11 & 8 & 10 & 5 & 1,348 \\
34 & El Corte Inglés & 7,21 & 8 & 10 & 1 & 2,100 \\
33 & Consum & 7,12 & 7 & 10 & 3 & 1,781 \\
30 & Estrella Levante & 8,30 & 8 & 10 & 5 & 1,179 \\
28 & Coato & 8,14 & 8 & 10 & 5 & 1,177 \\
24 & Inditex & 7,78 & 8 & 10 & 2 & 2,022 \\
19 & Zara & 7,32 & 8 & 10 & 4 & 1,668 \\
19 & Carrefour & 6,95 & 7 & 9 & 4 & 1,353 \\
18 & Cajamurcia & 5,89 & 6 & 10 & 1 & 2,720 \\
15 & Grupo Mondragón & 5,87 & 5 & 10 & 1 & 2,722 \\
10 & Telefónica & 5,80 & 6 & 9 & 3 & 2,251 \\
\hline
\end{tabular}

* En la agregación de valoraciones se buscó evitar duplicidad de menciones, incluyéndose en este cuadro únicamente las entidades que fueron mencionadas por más de 10 encuestados diferentes. Según los valores alcanzados, el nivel de reputación se podría calificar de: Excelente o superior (>8); Fuerte o robusta (7-7,9); Media o moderada (6-6,9); Débil o vulnerable (45,9); Débil o pobre $(<4)$.

FUENTE: Elaboración propia a partir de la Encuesta. 


\subsection{Dimensiones de la reputación racional de cooperativas y empresas}

Para alcanzar una medida del nivel de "reputación racional" de cada empresa, se planteaba otra pregunta, más extensa y compleja, en la que se le pedía a los encuestados que indicasen su nivel de acuerdo o de desacuerdo (según una escala de 1 a 10, indicando ese último estar totalmente de acuerdo), con una serie de afirmaciones que correspondían a la descripción de las siete dimensiones de la reputación que son planteadas por el modelo RepTrak, tal y como se ha descrito en el apartado de metodología.

A los encuestados se les planteaba que valorasen en cada uno de esos elementos a un par de empresas (una cooperativa versus empresa capitalista), ofreciéndoles la oportunidad de valorar tres posibles parejas (el hipermercado Eroski junto a Alcampo o Carrefour, el supermercado Consum junto a Mercadona; o bien a la entidad financiera Cajamar junto al Banco Santander) o en su caso, entre las dos empresas que eligiesen. Para simplificar los datos obtenidos se agruparon las organizaciones mencionadas en tres grandes categorías sectoriales: supermercados e hipermercados; entidades financieras y bancos; y otros, que incluía a empresas/cooperativas de sectores diversos (Cuadro 7). Finalmente, $y$ dado que hubo encuestados que indicaron no saber valorar esas dimensiones (de ninguna de las empresas ofrecidas, o en algún caso, sí poderlo hacer pero sólo para una de las dos propuestas), se contaba finalmente con la valoración de un total de 304 empresas cooperativas: en su mayor parte (78\%) pertenecientes a cadenas de distribución minorista; un $17 \%$ al sector bancario y un $5 \%$ a otros tipos de empresas. De igual modo, se obtuve la respuesta de un total de 307 encuestados que valoraron otros tipos de empresas (diferentes a las cooperativas), siendo igualmente mayoritario el grupo de Super-Hipermercados (75\%), con un $19 \%$ de bancos y un $6 \%$ de otras empresas.

Una primera aproximación a la valoración que los encuestados dieron a cooperativas y empresas era aportada por la pregunta en las que se pedía indicasen su nivel de acuerdo con la afirmación de que la empresa evaluada le "genera un alto nivel de confianza, respeto, admiración, estima o buena impresión". Esta pregunta, descrita en el apartado previo, tenía por objeto recabar la valoración que cada encuestado daba a las empresas atendiendo a una valoración de su "reputación emocional". Tal y como se muestra en las últimas filas del Cuadro 7, las cooperativas presentan una menor valoración media que otros tipos de empresas capitalistas, que en cualquier caso podría valorarse para ambos tipos de empresas de un nivel de reputación emocional "Medio o moderado" (6,33<6,72).

El nivel de reputación global se obtuvo agregando las siete dimensiones consideradas como media aritmética. Se comprueba nuevamente mediante este indicador agregado un nivel de reputación ligeramente menor en el caso de las cooperativas que de las empresas capitalistas $(6,11<6,32)$. Tanto en el caso de cooperativas como de las empresas capitalistas, son las entidades financieras (bancos) las que alcanzan una más baja reputación media (5,95 y 6,07 respectivamente), con una valoración ligeramente superior de Super-Hipermercados $(6,05$ y 6,26$)$, siendo superior a la media de cada grupo la 


\section{EL NIVEL DE CONOCIMIENTO Y LA REPUTACIÓN SOCIAL DE LAS EMPRESAS COOPERATIVAS. EL CASO DE LA REGIÓN DE MURCIA}

valoración que de manera específica reciben cooperativas $(7,64)$ y empresas capitalistas de "Otros sectores" $(7,74)$.

La revisión de las valoraciones medias otorgadas a cada una de las siete dimensiones, descritas en el Cuadro 7, permite identificar algunas diferencias entre tipos de empresas (cooperativa versus empresa capitalista). En todas las dimensiones consideradas, el análisis de la varianza realizado permite comprobar la significatividad de las diferencias de medias entre los sectores de actividad.

La primera de las ideas sería cómo las empresas capitalistas alcanzan una más elevada valoración en varias dimensiones: en Rentabilidad (D7), siendo más elevada su valoración (7,37>6,38 para cooperativas) de que estas tienen buenos resultados financieros y potencial de crecimiento; en Calidad de la oferta (D1), entendiendo los encuestados que es más cierto que las empresas capitalistas ofrecen productos y servicios de calidad, productos excelentes y servicios fiables, con una buena relaciónprecio y que satisface las necesidades de los clientes (6,93>6,76 para cooperativas); en Liderazgo (D6), valorando ligeramente por encima $(6,71>6,44)$ la buena organización y gestión profesional de las empresas capitalistas; en Grado de innovación (D2), otorgando una mayor valoración a las empresas capitalistas $(6,39>5,76)$, comprendiendo por tanto que son organizaciones más innovadoras; por último, en Trato de empleados (D3), siendo ligeramente superior la valoración media (5,79 para empresas capitalistas y 5,77 para cooperativas) por la que juzgan si son un lugar atractivo para trabajar.

Por el contrario, las sociedades cooperativas superan a las capitalistas en tan sólo dos elementos o dimensiones, como son: primero, la Ética en su gestión (D4), entendido los encuestados que estas responden en mayor medida a una gestión responsable, comprometiéndose de forma ética y mostrando una operativa transparente (5,88>5,64 para empresas capitalistas); y segundo, en Preocupación por la sociedad (D5), considerando los ciudadanos que estas se preocupan en mayor medida por la sociedad, apoyando buenas causas y procurando no dañar el medio ambiente $(5,77>5,38)$. 


\section{Cuadro 7. Comparación por sectores entre cooperativas y otras empresas según las dimensiones de reputación}

\begin{tabular}{|c|c|c|c|c|c|c|c|c|}
\hline \multirow[t]{2}{*}{ Tipo de empresas } & \multicolumn{4}{|c|}{ Empresas Cooperativas } & \multicolumn{4}{|c|}{ Empresas Capitalistas } \\
\hline & Super/Hiper & Bancos & Otros & Total & Super/Hiper & Bancos & Otros & Total \\
\hline Número de empresas (Porcentaje) & $\begin{array}{c}237 \\
(77,96)\end{array}$ & $\begin{array}{c}52 \\
(17,11)\end{array}$ & $\begin{array}{c}15 \\
(4,93)\end{array}$ & $\begin{array}{c}304 \\
(100,00)\end{array}$ & $\begin{array}{c}231 \\
(75,14)\end{array}$ & $\begin{array}{c}58 \\
(18,89)\end{array}$ & $\begin{array}{c}18 \\
(5,86)\end{array}$ & $\begin{array}{c}307 \\
(100,00)\end{array}$ \\
\hline $\begin{array}{l}\text { Valoración media de las dimensiones } \\
\text { de reputación racional* }\end{array}$ & Super/Hiper & Bancos & Otros & Total & Super/Hiper & Bancos & Otros & Total \\
\hline $\begin{array}{l}\text { D1: Ofrece productos y servicios de calidad, } \\
\text { productos excelentes y servicios fiables, de buena } \\
\text { relación-precio y satisface las necesidades de los clientes }\end{array}$ & 6,76 & 6,25 & 8,47 & 6,76 & 6,95 & 6,36 & 8,50 & 6,93 \\
\hline $\begin{array}{l}\text { D2: Es una empresa innovadora, ofreciendo } \\
\text { productos o servicios novedosos o innovando en } \\
\text { la manera de hacer las cosas } 2\end{array}$ & 5,74 & 5,42 & 7,27 & 5,76 & 6,33 & 6,14 & 7,94 & 6,39 \\
\hline $\begin{array}{l}\text { D3: Es un lugar atractivo para trabajar, con } \\
\text { buen trato al empleado y sueldos justos }\end{array}$ & 5,66 & 5,88 & 7,00 & 5,77 & 5,68 & 5,74 & 7,39 & 5,79 \\
\hline $\begin{array}{l}\text { D4: Es gestionada con responsabilidad, } \\
\text { comportándose de forma ética y que muestra } \\
\text { su operativa con transparencia } 4\end{array}$ & 5,78 & 5,88 & 7,53 & 5,88 & 5,64 & 5,26 & 6,83 & 5,64 \\
\hline $\begin{array}{l}\text { D5: Esa empresa se preocupa por la sociedad, } \\
\text { apoya buenas causas y procura no dañar el } \\
\text { medio ambiente }{ }^{5}\end{array}$ & 5,69 & 5,65 & 7,47 & 5,77 & 5,28 & 5,19 & 7,33 & 5,38 \\
\hline $\begin{array}{l}\text { D6: Es una compañía bien organizada, } \\
\text { liderada y gestionada con profesionalidad }\end{array}$ & 6,38 & 6,29 & 7,87 & 6,44 & 6,71 & 6,34 & 7,78 & 6,71 \\
\hline $\begin{array}{l}\text { D7: Esa empresa es una compañía rentable, } \\
\text { con buenos resultados financieros y que tiene } \\
\text { potencial de crecimiento } 7\end{array}$ & 6,31 & 6,25 & 7,87 & 6,38 & 7,26 & 7,47 & 8,44 & 7,37 \\
\hline Índice de Reputación Global (Media aritmética) ${ }^{8}$ & 6,05 & 5,95 & 7,64 & 6,11 & 6,26 & 6,07 & 7,74 & 6,32 \\
\hline Pro-Memoria & & mpresas C & perativas & & & mpresas $C$ & pitalistas & \\
\hline $\begin{array}{l}\text { Reputación emocional. Esa empresa me genera } \\
\text { un alto nivel de confianza, respeto, admiración, } \\
\text { estima o buena impresión } 9\end{array}$ & 6,33 & 6,37 & 7,80 & 6,41 & 6,72 & 6,52 & 8,33 & 6,78 \\
\hline $\begin{array}{l}\text { Mi grado de conocimiento de la empresa es } \\
\text { muy alto } 10\end{array}$ & 4,86 & 5,10 & 6,33 & 4,97 & 5,39 & 5,33 & 6,89 & 5,47 \\
\hline
\end{tabular}

Test $F$ de diferencia de medias de empresas cooperativas según sectores: ${ }^{1}(10,47 ; \alpha=0,000) ;{ }^{2}(6,50 ; \alpha=0,002) ;{ }^{3}(4,27 ; \alpha=0,015) ;{ }^{4}(6,93 ; \alpha=0,001)$; ${ }^{5}(6,09 ; \alpha=0,003) ;{ }^{6}(5,97 ; \alpha=0,003) ;{ }^{7}(6,51 ; \alpha=0,002) ;{ }^{8}(6,68 ; \alpha=0,004) ;{ }^{9}(5,07 ; \alpha=0,007) .{ }^{10}(4,47 ; \alpha=0,012)$.

Test $F$ de diferencia de medias en tipos de empresas capitalistas según sectores: ${ }^{1}(11,82 ; \alpha=0,000) ;{ }^{2}(5,96 ; \alpha=0,003) ;{ }^{3}(7,00 ; \alpha=0,001) ;{ }^{4}(4,72 ; \alpha=0,010)$; ${ }^{5}(9,36 ; \alpha=0,000) ;{ }^{6}(4,21 ; \alpha=0,016) ;{ }^{7}(3,58 ; \alpha=0,029) ;{ }^{8}(6,66 ; \alpha=0,008) ;{ }^{9}(6,31 ; \alpha=0,002) .{ }^{10}(4,51 ; \alpha=0,012)$.

*Según los valores alcanzados, el nivel de reputación se podría calificar de: Excelente o superior (>8); Fuerte o robusta (7-7,9); Media o moderada (6-6,9); Débil o vulnerable (4-5,9); Débil o pobre (<4).

FUENTE: Elaboración propia de la Encuesta. 


\section{Figura 1. Valoración de las 7 dimensiones de la reputación (cooperativas vs empresa capitalista)}

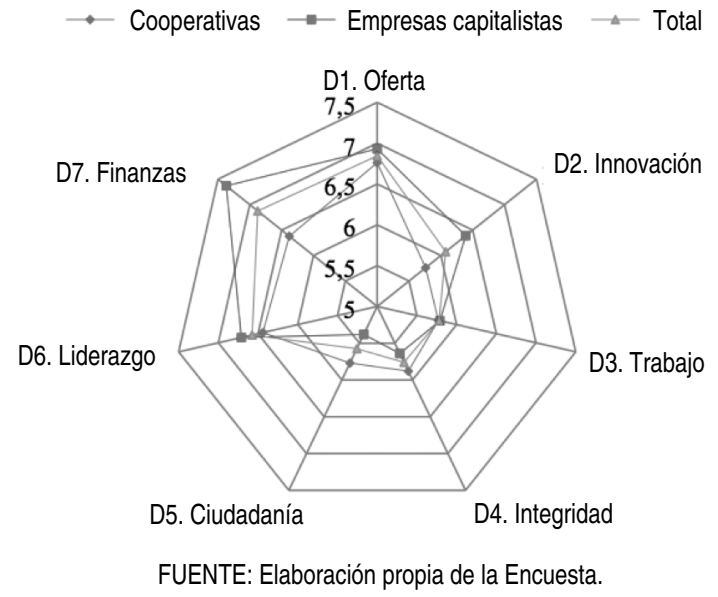

Para finalizar este epígrafe, y con objeto de hacer más visibles los elementos que definen la reputación de las empresas y que se describieron en el Cuadro 7, se presenta en la Figura 1 una representación radial de los resultados medios obtenidos por empresas cooperativas y capitalistas, para cada dimensión, confrontándose ambos modelos. Así, para las cooperativas las tres dimensiones más valoradas son Calidad de la oferta (D1), seguida de Liderazgo (D6) y Rentabilidad (D7), con valores medios de 6,76, 6,44 y 6,38 respectivamente; seguidas por Ética en su gestión (D4), Preocupación por la sociedad (D5), Trato de empleados (D3) y Grado de innovación (D2). En cuanto a la media de las 7 dimensiones de las empresas capitalistas, sus más elevadas valoraciones se alcanzan en Rentabilidad (D7), Calidad de la oferta (D1) y Liderazgo (D6), con valoraciones medias de 7,37, 6,93 y 6,71 respectivamente. En cuarto lugar vendría el Grado de innovación (D2), seguida del Trato de empleados (D3), Ética en su gestión (D4) y Preocupación por la sociedad (D5).

Para el total de los datos, incluyendo tanto a las empresas cooperativas como a las capitalistas, se puede comprobar que eran la Rentabilidad (D7), la Calidad de la oferta (D1) y el Liderazgo (D6) las 3 dimensiones más valoradas por los encuestados, seguidas por el Grado de la innovación (D2), el Trato a empleados (D3), la Ética en su gestión (D4) y la Preocupación por la sociedad (D5).

Por tipos de entidad, tan sólo las empresas capitalistas alcanzan una dimensión (D7: Esa empresa es una compañía rentable...) en la que su valoración media supera la valoración de 7, equivalente a un nivel de reputación "Fuerte o robusto". Por su parte las cooperativas se encuentran en niveles de reputación "Media o moderada" (puntuaciones entre 6 y 6,9) en tres de sus dimensiones: (D1) Ofrece productos y servicios de calidad,...; (D6) Es una compañía bien organizada...; y (D7) Esa empresa 
es una compañía rentable, con buenos resultados...). Su nivel de reputación es "Débil o vulnerable" (entre 4 y 5,9) en cuatro dimensiones (D2: Es una empresa innovadora...; D3: Es un lugar atractivo para trabajar...; y D4: Es gestionada con responsabilidad, comportándose de forma ética y que muestra su operativa con transparencia), poniendo en entredicho algunos de los principios que se esperaría fuesen reconocibles socialmente en las cooperativas.

\section{Conclusiones}

El análisis de la encuesta realizada a ciudadanos de la Región de Murcia ha permitido comprobar el alto nivel de confianza que frente a grandes empresas tienen las PYME's y cooperativas, siendo incluso superior a la calificación que otorgan a ONGs. En términos de conocimiento, una gran parte indica saber qué es una cooperativa, relacionándolo con un "modelo de empresa", si bien existe -aunque sea muy escaso- una creencia o confusión acerca de si en todos los casos son Organizaciones Sin Fines de Lucro. Se obtuvo también que la mayoría de los encuestados han escuchado alguna vez noticias sobre las cooperativas; además, han sabido mencionar alguna cooperativa, afirmando que en alguna ocasión consumieron un producto y/o servicio procedente de las mismas. De otro lado, una pequeña parte es -o ha sido- socio de alguna cooperativa, siendo por el contrario alto el número de ciudadanos que han dicho conocer a alguien que es socio o que trabaja en una cooperativa, siendo por tanto diversas las relaciones y múltiples las fuentes de conocimiento que los ciudadanos tienen del modelo cooperativo.

La imagen de las cooperativas ha resultado ser bien valorada en comparación con las empresas capitalistas, en especial en su contribución al "desarrollo de las zonas rurales", la "democracia interna" y su "profesionalidad", pudiendo "competir en igualdad de condiciones" con otras empresas. Ese análisis se ha completado con la información generada sobre la reputación de las cooperativas, que aporta información muy relevante acerca de sus fortalezas y debilidades en opinión de los ciudadanos. Se ha podido comprobar cómo el nivel de reputación que en general tienen las cooperativas es "Débil" o "Vulnerable", e inferior globalmente al que alcanzan las empresas capitalistas. Además, se han identificado dimensiones en las que es preciso que las cooperativas aumenten sus esfuerzos competitivos, como puede ser la Innovación o la Calidad del producto. La reputación social de las cooperativas sólo sobresale en dos dimensiones, relativas a un comportamiento más ético (Responsabilidad) y a una mayor preocupación por la sociedad (Ciudadanía). Por el contrario, no se produce una mayor reputación de las cooperativas -frente al de las empresas capitalistas- en otros ámbitos, como pudieran ser en su capacidad de generar condiciones de trabajo de calidad, siendo urgente que las empresas cooperativas recuperen el reconocimiento de algunos de los principios cooperativos que las identifica y diferencia. 


\section{Referencias bibliográficas}

ACI (2013): Proyecto de una Década Cooperativa, Alianza Cooperativa Internacional (ACI), pp. 32.

AGIRRE, A. (2001): "Los principios cooperativos "atractores" de la gestión eficiente: su medición. Aplicación al caso de Mondragón Corporación Cooperativa", CIRIEC-España, Revista de Economía Pública, Social y Cooperativa, 39, 93-113.

ALAMEDA, A.P., OLARTE, P.C., REINARES, L.E.M. \& SACO, V.M. (2006): "Notoriedad de marca y medios de comunicación", ESIC Market, 124, 91-116.

BIRCHALL, J. \& SIMMONS, R. (2013): Resilience in a Downturn: the power of financial cooperatives, Geneva: International Labour office.

BORRAZ, M.J. \& FUENTELSAZ, L.L. (2005): La gestión del ciclo de vida de las capacidades: un análisis para el caso de la reputación, IV Iberoamerican Academy of Management, Lisboa.

COMISIÓN EUROPEA (2010): Eurobarómetro 74. Opinión pública en la Unión Europea, informe nacional España, Disponible en: http://ec.europa.eu/spain/pdf/eurobarometro_74_espana.pdf

CONCOVAL (2008): Imagen del Cooperativismo en la Comunidad Valenciana 2007, Confederació de Cooperatives de la Comunitat Valenciana (CONCOVAL). Disponible en: http://www.concoval.es (consultado el 05-07-2017).

DEFOURNY, J. \& NYSSENS, M. (2012): "Social Cooperatives: When Social Enterprise Meets the Cooperative Tradition", Paper presented at the Euricse Conference in Venice.

EID, M. \& MARTÍNEZ-CARRASCO PLEITE, F. (2014): "El año internacional de las cooperativas. Una aproximación a los desafíos del sector mediante el método Delphi”, REVESCO, Revista de Estudios Cooperativos, 116, 103-129.

FOMBRUN, C.J. (1996): Reputation: Realising Value from the Corporate Image, Harvard Business School Press, Boston.

GROENLAND, E.A. (2002): "Qualitative Research to Validate RQ-Dimensions", Corporate Reputation Review, 4(4), 308-315.

MANDELLI, A. \& CANTONI, L. (2010): "Social media impact on corporate reputation: Proposing a new methodological approach", Cuadernos de Información, 27, 61-74.

MARCUELLO, C. \& SAZ, M.I. (2008): "Los principios cooperativos facilitadores de la innovación: un modelo teórico", REVESCO, Revista de Estudios Cooperativos, 94, 59-79. 
MARTÍNEZ, I.M. \& OLMEDO, I. (2010): "Revisión teórica de la reputación en el entorno empresarial", Cuadernos de Economía y Dirección de la Empresa, 44, 59-77.

MARTÍNEZ, I.M., OLMEDO, I. \& ZAPATA, J. (2013): "Reputación percibida por el profesorado de las cooperativas de educación: medición e influencia", CIRIEC-España, Revista de Economía Pública, Social y Cooperativa, 77, 237-260.

MARTÍNEZ-CARRASCO PLEITE, F. (Dir.)(2011): Las grandes siglas de la Economía Social de la Región de Murcia, Editum, Universidad de Murcia, 162 pp. Disponible en: http://libros.um.es/editum/catalog/book/301 (consultado el 02-06-2015).

MOREIRA, M. (1999): "A Globalização e o Movimento Cooperativo. Tópicos para o debate". En: 0 Cooperativismo no Novo Milénio. A Vantagem Cooperativa, Lisboa: INSCOOP - Instituto António Sérgio do Sector Cooperativo, 53-94.

MOZAS, A., MOYANO, J., SENISE, O., PARRAS, M. \& MURGADO, E.M. (2005): "Cultura cooperativa y eficiencia empresarial: Una contrastación en el cooperativismo oleícola", REVESCO, Revista de Estudios Cooperativos, 85, 41-60.

MTESS (2016): Avance - Resumen situación empresas de Economía Social y sus Trabajadores en situación de alta en la Seguridad Social, Ministerio de Empleo y Seguridad Social.

OIT (2012): La forma cooperativa de hacer negocios. El enfoque de la OIT para apoyar a las cooperativas, Organización Internacional del Trabajo (OIT), Servicio de Cooperativas (EMP/COOP).

OLMEDO, I., MARTíNEZ, M., ARCAS, N. \& MARÍN, L. (2012): "Relación circular entre ética, responsabilidad social y reputación de las cooperativas", REVESCO, Revista de Estudios Cooperativos, 107, 129-154.

ONU (2010): "Las cooperativas en el desarrollo social. Organización de Naciones Unidas. Resolución aprobada por la Asamblea General", [sobre la base del informe de la Tercera Comisión (A/64/432)], $64 / 136$.

ONU (2011): Report of the Expert Group Meeting Cooperatives in Social Development: Beyond 2012, 3-6 May Ulaanbaatar, Mongolia. Disponible en: http://www.un.org/esa/socdev/egms/docs/2011/coops-egm-report.pdf (consultado el 6/07/2014).

OROZCO, T.J.A. \& FERRÉ P.C. (2012): "Los índices de reputación corporativa y su aplicación en las empresas de comunicación", III congreso Asociación Española de Investigación de la Comunicación, Tarragona.

PÉREZ, A. \& RODRÍGUEZ DEL BOSQUE, I. (2014): "Identidad, imagen y reputación de la empresa: integración de propuestas teóricas para una gestión exitosa", Cuadernos de Gestión, 14(1), 94126.

PÉROTIN, V. (2012): "Workers' Cooperatives: Good, Sustainable Jobs in the Community", Journal of Entrepreneurial and Organizational Diversity, 2, 34-47. 
PUZINO, S.L. (2009): "Deuda, desarrollo local y economía social", CIRIEC-España, Revista de Economía Pública, Social y Cooperativa, 64, 35-58.

REPUTATION INSTITUTE (2013): Informe RepTrak Pulse España 2013, Disponible en: www.reputationinstitute.es (consultado el 05-04-2013).

REPUTATION INSTITUTE (2014): Informe RepTrak Pulse España 2014, Disponible en: www.reputationinstitute.es (consultado el 05-07-2014).

ROSSITER, J.R. \& PERCY, L. (1987): Advertising and Promotion Management, Mc Graw-Hill: New York.

SANZ, M. \& GONZÁLEZ, M. (2005): Identidad Corporativa. Claves de la comunicación empresarial, Madrid: ESIC.

VARGAS, A. (1995): "La identidad cooperativa y la cooperativa como empresa: luces y sombras", REVESCO, Revista de Estudios Cooperativos, 61, 179-192.

VIDAL, M.I. (2008): “¿Qué es la economía social?”, Revista de Cooperativismo Sanitario, 72, 22-25.

WESSELS, C. (2003): "Reputation defined", CSR Magazine, 2/03, 28-29. 
